\title{
Learning and the behavior of potential entrants
}

\author{
Gautam Bhattacharya*
}

The possibility of cost reduction through learning by gathering experience is important in many industries where organizational efficiency in production and management is an important determinant of costs. In these industries, the difference in unit costs of production between experienced firms and potential entrants retards entry. This article investigates the behavior of a potential entrant in such an industry and the nature of dynamic equilibria resulting from the interaction of the entrant and the current producer.

\section{Introduction}

The process of cost reduction through learning from experience is important in many industries where organizational efficiency in production and management can significantly alter costs. ${ }^{1}$ Because of learning effects, potential entrants in these industries anticipate that they will initially face a higher cost structure than the incumbent firms, and this can retard entry. Here, one possible strategy for the potential entrant is to adopt a learning process beginning before entry and continuing afterwards-for example, by starting a small-scale experimental firm or by selling to a small group of consumers or even by acquiring experience about production technology through $R \& D$. Although such a strategy loses money in the beginning, its learning effects enable the entrant to enter actively if costs are sufficiently reduced and to obtain higher profits from cost-reductions occurring after entry. This article analyzes the entrant's process of decisionmaking about a cost-reducing learning strategy and examines the pattern of entry and the nature of the industry equilibrium after entry in the context of a stochastic dynamic game. It also examines how industry equilibrium, entry patterns, and learning strategies are affected if there are exogenous changes in the initial cost disadvantage, in technology, in the discount rate, and in other parameters. Unlike Spence (1981), this article considers entry to be endogenous rather than exogenous. Further, in Spence cost reductions through learning depend on cumulative output and do not involve any uncertainty. In this article the learning process does not depend on cumulative output, but is characterized as a continuous-time stochastic Poisson process (with the probability of success taken as a decision variable); each success is assumed to have a

\footnotetext{
* University of Kansas.

I am grateful to the Editorial Board, A. Dixit, and two anonymous referees for their comments and suggestions. However, I alone am responsible for any remaining errors. An earlier version of this article was presented at the Econometric Society Winter Meeting in New York in December, 1982. Part of the research was supported by University of Kansas grant \#3029-0038.

' See Abell (1979), Buzacott (1981), Porter (1980), and Gold (1981) for a discussion of learning curves. See Zimmerman (1982) for a discussion of learning effects in the nuclear power industry.
} 
fractional reduction effect on the unit-costs of the entrant's production. Section 2 develops the model and Section 3 discusses the nature of dynamic equilibrium.

\section{Dynamic equilibrium}

The industry considered has two firms-a monopolist and a potential entrant. The monopolist has a constant unit cost of production $\alpha$. The potential entrant's initial unit cost is $\eta_{0}$, where $\eta_{0}=\alpha+\delta+\beta, \delta \geq 0$, and $\beta>0$. The initial cost difference $(\beta+\delta)$ exists because through a long period of involvement in the production process, the monopolist has acquired sufficient experience to choose the best technology and organization. Consequently, the monopolist is assumed to have no further scope for cost reduction. The entrant, however, may choose to adopt a learning process before entry through which he will gather experience, possibly reduce costs, and enter as an active competitor when costs are sufficiently lowered. This activity is characterized by a continuous-time Poisson process of successful cost reductions ("successes") when the probability of one success at each instant of time is $\gamma$, which is a decision variable. After $k$ such successes, the entrant's unit cost is

$$
\eta_{k}=\alpha+\delta+\beta \lambda^{k}, \quad 0<\lambda<1 .
$$

Thus every success reduces a part of the cost difference $\beta$ by a fraction $\lambda$. However, $\delta$ is the part of the cost difference that cannot be eliminated by the learning process (though $\delta$ can possibly be zero). It is assumed later than $\delta$ is sufficiently small so that entry would be profitable for any firm with a large enough number of successes (see (11)). The entrant's strategy is to choose $\gamma$ and output $x_{e}$ at every instant of time. Similarly, the monopolist chooses his output strategy $x_{m}$ at every instant of time. The following standard assumptions are made about the inverse demand function $P\left(x_{m}+x_{e}\right)$ and the total revenue functions $R_{m}=P x_{m}$ and $R_{e}=P x_{e}$ :

$$
\begin{array}{r}
P(0)=\bar{P}<\infty ; \quad P(\bar{x})=0 ; \quad \bar{x}<\infty ; \quad P^{\prime}<0 ; \quad P^{\prime \prime} \text { is continuous; } \\
\frac{\partial^{2} R_{m}}{\partial x_{m}^{2}}<0 ; \quad \frac{\partial^{2} R_{m}}{\partial x_{m} \partial x_{e}}<0 ; \quad \frac{\partial^{2} R_{e}}{\partial x_{e}^{2}}<0 ; \quad \frac{\partial^{2} R_{e}}{\partial x_{e} \partial x_{m}}<0 .
\end{array}
$$

I shall consider a subgame-perfect equilibrium ${ }^{2}$ for this dynamic stochastic game (Selten, 1975). Both players maximize the expected value of the sum of discounted profits over an infinite horizon ( $r$ is taken to be the discount rate). After the entrant has chosen $\gamma$ at the outset, however, there is a subgame at each instant $t$ where each maximizes his current profit, given the current unit costs of production of both players. Let $F(\gamma)$ be the instantaneous cost associated with $\gamma$ incurred at every $t$ with $F(0)=0$. It is assumed that the learning cost $F(\gamma)$ is increasing and convex in $\gamma\left(F^{\prime}>0, F^{\prime \prime}>0\right)$. Further, let $\bar{\gamma}<1$ be the fastest possible speed of learning the entrant can choose. If $\pi_{m}\left(x_{m}, x_{e}, \alpha, \eta_{t}\right)$ and $\pi_{e}\left(x_{m}, x_{e}, \alpha\right.$, $\eta_{t}$ ) are instantaneous profits of the monopolist and the potential entrant, respectively, then a subgame-perfect equilibrium with Cournot-Nash equilibrium for each subgame is defined as a pair of strategies $\left\{x_{m}^{*}\left(\alpha, \eta_{t}\right)\right\}$ and $\left\{\gamma^{*}, x_{e}^{*}\left(\alpha, \eta_{t}\right)\right\}$ such that for every $t$,

and

$$
\begin{gathered}
\pi_{m}\left(x_{m}^{*}\left(\alpha, \eta_{t}\right), x_{e}^{*}\left(\alpha, \eta_{t}\right), \alpha, \eta_{t}\right) \geq \pi_{m}\left(x_{m}, x_{e}^{*}\left(\alpha, \eta_{t}\right), \alpha, \eta_{t}\right), x_{m} \geq 0 \\
\pi_{e}\left(x_{m}^{*}\left(\alpha, \eta_{t}\right), x_{e}^{*}\left(\alpha, \eta_{t}\right), \alpha, \eta_{t}\right) \geq \pi_{e}\left(x_{m}^{*}\left(\alpha, \eta_{t}\right), x_{e}, \alpha, \eta_{t}\right), x_{e} \geq 0
\end{gathered}
$$

$$
\gamma^{*} \text { maximizes } E\left(\int_{0}^{\infty} e^{-r t}\left(\pi_{e}\left(x_{m}^{*}\left(\alpha, \eta_{t}\right), x_{e}^{*}\left(\alpha, \eta_{t}\right), \alpha, \eta_{t}\right)-F(\gamma)\right) d t \mid \gamma\right) ; 0 \leq \gamma \leq \bar{\gamma}
$$

where $E(\cdot \mid \gamma)$ denotes expectation with respect to the Poisson-process generated by $\gamma$.

${ }^{2}$ I am indebted to a referee for suggesting the use of subgame-perfect equilibrium instead of the usual openloop Nash equilibrium in this context. It should be noted, however, that each player's strategy in the equilibrium considered is dependent only on the current cost levels of both players and not on the past actions of the players. 
In this game the equilibria in the subgames played at each instant are the usual Cournot-Nash equilibria with the monopolist's unit cost $\alpha$ and the entrant's unit cost $\eta_{t}$ at time $t$. The following lemmas describe well-known properties of such equilibria (see Bhattacharya (1982) for proof of these lemmas).

Lemma 1. For every $\alpha, \eta$ such that $0 \leq \alpha \leq \bar{P}, 0 \leq \eta \leq \bar{P}$, there exists a unique CournotNash equilibrium $x_{m}^{*}(\alpha, \eta) \geq 0$ and $x_{e}^{*}(\alpha, \eta) \geq 0$.

Let the equilibrium profits be $\phi_{m}(\alpha, \eta)=\pi_{m}\left(x_{m}^{*}(\alpha, \eta), x_{e}^{*}(\alpha, \eta), \alpha, \eta\right)$ and

$$
\phi_{e}(\alpha, \eta)=\pi_{e}\left(x_{m}^{*}(\alpha, \eta), x_{e}^{*}(\alpha, \eta), \alpha, \eta\right) .
$$

Lemma 2. For any $\alpha \in[0, \bar{P})$, there exists $\eta^{*}(\alpha)$ such that

$$
\begin{array}{lllll}
x_{e}^{*}(\alpha, \eta)=0, & \phi_{e}(\alpha, \eta)=0 & \text { for } & \eta \geq \eta^{*}(\alpha), & \eta \leq \bar{P} \\
x_{e}^{*}(\alpha, \eta)>0, & \phi_{e}(\alpha, \eta)>0 & \text { for } & \eta<\eta^{*}(\alpha), & \eta \geq 0 .
\end{array}
$$

Further, if $\alpha<\eta<\eta^{*}(\alpha)$, then

$$
\begin{array}{rlrlrl}
\frac{\partial x_{e}^{*}}{\partial \alpha}>0, & \frac{\partial x_{e}^{*}}{\partial \eta}<0, & \frac{\partial x_{m}^{*}}{\partial \alpha}<0, & & \frac{\partial x_{m}^{*}}{\partial \eta}>0 \\
\frac{\partial \phi_{e}}{\partial \alpha}>0, & \frac{\partial \phi_{e}}{\partial \eta}<0, & \frac{\partial \phi_{m}}{\partial \alpha}<0, & \frac{\partial \phi_{m}}{\partial \eta}>0 .
\end{array}
$$

As an example of the model to be developed in the next section, the case of linear demand curves will be considered. The relevant information about static Cournot equilibrium in the case of linear demand curves is given below.

Let $P=\bar{P}-b\left(x_{m}+x_{e}\right)$. Let $\alpha<\bar{P}$. Then

$$
\begin{gathered}
\phi_{e}(\alpha, \eta)=\frac{1}{9 b}(\bar{P}+\alpha-2 \eta)^{2}, \quad 0<\eta<\frac{\bar{P}+\alpha}{2} \\
\phi_{e}(\alpha, \eta)=0, \quad \eta \geq \frac{\bar{P}+\alpha}{2} \\
\phi_{m}(\alpha, \eta)=\frac{1}{9 b}(\bar{P}+\eta-2 \alpha)^{2} \\
\eta^{*}(\alpha)=\frac{\bar{P}+\alpha}{2} .
\end{gathered}
$$

From Lemma 2 it can be seen that the firm will enter as an active producer if its unit cost is sufficiently reduced and falls below $\eta^{*}(\alpha)$. Assume that $k^{*}$ successful cost reductions through learning are necessary to enter actively; that is, there exists a positive integer $k^{*}$ such that

$$
\eta_{k^{*}}=\alpha+\delta+\beta \lambda^{k^{*}}<\eta^{*}(\alpha)<\alpha+\delta+\beta \lambda^{k^{*-1}}=\eta_{k^{*}-1} .
$$

Let $t_{k}$ be the time taken to achieve $k$ successes when $\gamma$ is the probability of one successful cost reduction at any instant of time.

Lemma 3. $t_{k}$ is a random variable which has a gamma distribution with parameters $1 / \gamma$ and $k$.

Proof. If $k(t)$ is the number of successes up to time $t$, then

$$
\operatorname{Prob}(k(t+h)-k(t)=1 \mid k(t)=k)=\gamma h+0(h), \quad k=0,1,2 .
$$

It is shown in Karlin and Taylor (1975) and Bartlett (1978) that if the waiting time between the $(k-1)$ th and $k$ th jumps is $s_{k}=t_{k}-t_{k-1}, k=1,2,3, \ldots$, then $s_{k}$ 's are 
independent and identically distributed as exponential variates with parameter $1 / \gamma$. The distribution of $t_{k}$ will therefore be the joint density of $k$ of $s_{k}$ 's, and hence will be the gamma distribution with parameters $(1 / \gamma, k)$. If $g\left(t_{k} \mid \gamma\right)$ is the probability density function of $t_{k}$, then

$$
g\left(t_{k} \mid \gamma\right)=\frac{\gamma}{(k-1) !} e^{-\gamma t_{k}}\left(\gamma t_{k}\right)^{k-1}, \quad 0 \leq t_{k}<\infty . \quad \text { Q.E.D. }
$$

Since $k^{*}$ is the required number of cost reductions through learning before the entrant can enter actively, from Lemma 3 the expected entry time $E\left(t_{k^{*}}\right)$ is $k^{*} / \gamma^{*}$ and the variance of $t_{k^{*}}$ is $k^{*} / \gamma^{2}$. The expected entry time therefore varies directly with the number of times costs have to be reduced before the entrant can make a positive profit. It also varies inversely with the speed of learning $\gamma$.

To prove that a subgame-perfect equilibrium exists, the expected total profits of the entrant will be expressed as a function of $\gamma$. For any chosen $\gamma$, the entrant's unit cost between $t_{k}$ and $t_{k+1}$ is $\eta_{k}=\alpha+\beta \lambda^{k}+\delta$. If $k<k^{*}$, his instantaneous profit is zero (as he has not entered yet). If $k \geq k^{*}$, then his instantaneous profit is $\phi_{e}\left(\alpha, \eta_{k}\right)$ between intervals $\left[t_{k}, t_{k+1}\right]$, assuming Cournot-Nash equilibrium occurs in each instantaneous subgame. Hence his expected discounted total profit is evaluated as

$$
\begin{aligned}
W_{e}(\gamma) & =-\int_{0}^{\infty} F(\gamma) e^{-n} d t+E\left(\sum_{j=0}^{\infty} \int_{t k^{*}+j}^{t k^{*}+j+1} \phi_{e}\left(\alpha, \eta_{k^{*}+j}\right) e^{-r t} d t\right) \\
& =-\frac{F(\gamma)}{r}+\frac{1}{r} E\left(\sum_{j=0}^{\infty} \phi_{e}\left(\alpha, \eta_{k^{*}+j}\right)\left(e^{-r k^{*}+j}-e^{-r k^{*}+j+1}\right)\right. \\
& =-\frac{F(\gamma)}{r}+\frac{\gamma^{k^{*}}}{(\gamma+r)^{k^{*}}} \sum_{j=0}^{\infty} \frac{\gamma^{j}}{(r+\gamma)^{j+1}} \phi_{e}\left(\alpha, \eta_{k^{*}+j}\right),
\end{aligned}
$$

as $t_{k^{*}+j}$ is a gamma variate with parameters $\left(1 / \gamma, k^{*}+j\right)$.

Hence $W_{e}(\gamma)$ is a continuous function of $\gamma$, and a maximum exists over the compact range $[0, \bar{\gamma}]$ at $\gamma^{*}$. Therefore, it has been shown that a subgame-perfect equilibrium exists with equilibrium strategies $\left\{\gamma^{*},\left\{x_{e}^{*}\left(\alpha, \eta_{t}\right)\right\}\right.$ for the entrant and $\left\{x_{m}^{*}\left(\alpha \eta_{t}\right)\right\}$ for the monopolist.

\section{Displacements of the dynamic equilibrium}

- From (14),

$$
W_{e}(\gamma)=-\frac{F(\gamma)}{r}+\sum_{j=k^{*}}^{\infty} \phi_{e}\left(\alpha, \eta_{j}\right) \gamma^{j} /(r+\gamma)^{j+1}
$$

Obviously, the first term on the right-hand side of (15) is the present value of the stream of learning costs, while the second term is the present value of the stream of expected profits starting from time $t_{k^{*}}$ when $k^{*}$ successes have been achieved. If $W_{e}(\gamma)$ is a concave function of $\gamma$, then the optimum $\gamma^{*}$ is unique. However,

$$
\begin{aligned}
& W_{e}^{\prime}(\gamma)=-\frac{F^{\prime}(\gamma)}{r}+\sum_{j=k^{*}}^{\infty} \phi_{e}\left(\alpha, \eta_{j}\right) \frac{\gamma^{j-1}(j r-\gamma)}{(r+\gamma)^{j+2}} \\
& W_{e}^{\prime \prime}(\gamma)=-\frac{F^{\prime \prime}(\gamma)}{r}+\sum_{j=k^{*}}^{\infty} \phi_{e}\left(\alpha, \eta_{j}\right) \frac{\gamma^{j-2}}{(r+\gamma)^{j+3}}\left[j(j-1) r^{2}-4 j r \gamma+2 \gamma^{2}\right]
\end{aligned}
$$

As $F(\gamma)$ is convex, $F^{\prime \prime}(\gamma)>0$. Therefore, $W_{e}^{\prime \prime}(\gamma)$ is negative if

$$
\left(j(j-1) r^{2}-4 j r \gamma+2 \gamma^{2}\right)<0
$$

or

$$
\gamma / r \in\left(j-[j(j+1) / 2]^{1 / 2}, j+[j(j+1) / 2]^{1 / 2}\right), \quad j=k^{*}, k^{*}+1, \ldots
$$

Hence, it is obvious that $W_{e}(\gamma)$ may not be concave, and multiple optima may exist. 
To simplify the characterization of equilibrium, it is assumed that

$$
W_{e}^{\prime \prime}\left(\gamma^{*}\right) \neq 0 \text {. }
$$

If (18) holds, three types of equilibria may exist (multiple equilibria may also exist):

(a) $\gamma^{*}=0$. As $k^{*}>0$, the entrant's expected profits cannot be positive if $\gamma^{*}=0$. So $\gamma=0$ is optimal only if $W_{e}(\gamma) \leq 0,0 \leq \gamma \leq \bar{\gamma}$. Here $t_{k^{*}}=\infty$ or the potential entrant decides not to initiate a learning process because learning costs are excessive or expected postentry profits are insufficient.

(b) $\gamma^{*}=\bar{\gamma}$ or the speed of learning chosen is the highest possible one available to the firm. Here $W_{e}^{\prime}(\bar{\gamma}) \geq 0$.

(c) $0<\gamma^{*}<\bar{\gamma}$. This is the case of an interior optimum. Here

$$
\begin{gathered}
\frac{F^{\prime}\left(\gamma^{*}\right)}{r}=\sum_{j=k^{*}}^{\infty} \phi_{e}\left(\alpha, \eta_{j}\right) \frac{\gamma^{* j-1}\left(j r-\gamma^{*}\right)}{\left(r+\gamma^{*}\right)^{j+2}}, \\
W_{e}^{\prime \prime}\left(\gamma^{*}\right)<0
\end{gathered}
$$

Equation (19) shows that in case of an interior optimum, marginal cost of the speed of learning $\gamma^{*}$ should be equal to addition to expected present value of postentry profits from lower costs and from earlier entry-time resulting from a faster speed of learning. Equation (19) will be used extensively to characterize the nature of dynamic equilibrium in the rest of this section, assuming that the optimum $\gamma^{*}$ is an interior optimum.

First, consider the effect of a larger initial difference between the unit-costs of the monopolist and the entrant. At first glance, it seems that this will lead to a new dynamic equilibrium with a slower speed of learning adopted by the entrant. As seen below, however, this is not necessarily true.

Let a higher cost difference be reflected by a higher $\delta$. If the change in $\delta$ is small, $k^{*}$ (as defined by (11)) will not change, but the entrant's cost at entry time and in all subsequent periods $\left(\alpha+\delta+\lambda^{k^{*}} \beta\right)$ will be higher. The effect of a change in $\delta$ on the entrant's speed of learning in dynamic equilibrium is found as

$$
\frac{d \gamma^{*}}{d \delta}=-\frac{1}{W_{e}^{\prime \prime}\left(\gamma^{*}\right)}\left(\frac{\partial}{\partial \delta} W_{e}^{\prime}\left(\gamma^{*}\right)\right)
$$

$W_{e}^{\prime}\left(\gamma^{*}\right)$, however, can be rewritten as

$$
\begin{aligned}
W_{e}^{\prime}\left(\gamma^{*}\right)=-\frac{F^{\prime}(\gamma)}{r} & +\sum_{j=0}^{\infty} \phi_{e}\left(\alpha, \eta_{k^{*}+j}\right) \frac{k^{*} r \gamma^{k^{*}+j-1}}{(r+\gamma)^{k^{*}+j+2}} \\
& +\sum_{j=0}^{\infty} \phi_{e}\left(\alpha, \eta_{k^{*}+j}\right) \frac{\gamma^{k^{*}}}{(r+\gamma)^{k^{*}}}\left(\frac{j \gamma^{j-1}}{(r+\gamma)^{j+1}}-\frac{(j+1) \gamma^{j}}{(r+\gamma)^{j+2}}\right), \quad \gamma=\gamma^{*} .
\end{aligned}
$$

Differentiating with respect to $\delta$, writing $\phi_{e \eta}=\partial \phi_{e} / \partial \eta=\partial \phi_{e} / \partial \delta$, and rearranging the second term on the right-hand side of $(16 \mathrm{a})$, we get

$$
\begin{aligned}
& \frac{\partial W_{e}^{\prime}\left(\gamma^{*}\right)}{\partial \delta}=\sum_{j=0}^{\infty} \phi_{e \eta}\left(\alpha, \alpha+\delta+\lambda^{k^{*}+j} \beta\right) \frac{k^{*} r \gamma^{k^{*}+j-1}}{(r+\gamma)^{k^{*}+j+2}} \\
& +\sum_{j=0}^{\infty}(j+1) \gamma^{j}(r+\gamma)^{-j-2}\left[\phi_{e \eta}\left(\alpha, \alpha+\delta+\lambda^{k^{*}+j+1} \beta\right)-\phi_{e \eta}\left(\alpha, \alpha+\delta+\lambda^{k^{*}+j} \beta\right)\right], \quad \gamma=\gamma^{*} .
\end{aligned}
$$

From Lemma $2 \phi_{e \eta}<0$. If $\phi_{e}$ is convex in $\eta$, then each term on the right-hand side of (22) is negative and from (21) $d \gamma^{*} / d \delta<0$. On the other hand, if $\phi_{e}$ is concave in $\eta$, then it can be shown that $d \gamma^{*} / d \delta>0$ if 
and

$$
\left|\frac{\phi_{e_{\eta}}\left(\alpha, \alpha+\delta+\lambda^{k^{*}+j+1} \beta\right)-\phi_{e \eta}\left(\alpha, \alpha+\delta+\lambda^{k^{*}+j} \beta\right)}{\phi_{e \eta}\left(\alpha, \alpha+\delta+\lambda^{k^{*}+j} \beta\right)}\right| \geq \frac{r k^{*}}{\gamma^{*}}, \quad j=0,1, \ldots
$$

We therefore have:

Proposition 1. If postentry equilibrium profit is a convex function of the entrant's unit cost, then an increase in the initial cost difference between the monopolist and the entrant will reduce the speed of learning adopted by the entrant. On the other hand, if postentry proft is concave and the proportional fall in marginal profits from any successive cost reductions is bounded below by $r k^{*} / \gamma^{*}$, then an increase in initial cost difference will increase the speed of learning of the entrant.

In the case of straight-line demand curves, it can be checked from (10a) that $\phi_{e}$ is convex in $\eta$; hence $d \gamma^{*} / d \delta<0$.

Other effects of an increase in $\delta$ depend on the sign of $d \gamma^{*} / d \delta$. If $\gamma^{*}$ falls as $\delta$ rises, the expected entry time $k^{*} / \gamma^{*}$ increases: entry occurs later on the average. $\operatorname{Var}\left(t_{k^{*}}\right)$ or the variance of entry time also rises. Further, expected total postentry profits will also fall in this case. On the other hand, if $d \gamma^{*} / d \delta>0$, then both $E\left(t_{k^{*}}\right)$ and $\operatorname{var}\left(t_{k^{*}}\right)$ fall as $\delta$ rises.

Finally, the expected present value of profits $W_{e}\left(\gamma^{*}\right)$ will always fall if $\delta$ rises irrespective of the sign of $d \gamma^{*} / d \delta$. This can be verified from the envelope theorem:

$$
\begin{aligned}
\frac{d}{d \delta} W_{e}\left(\gamma^{*}\right) & =\frac{\partial}{\partial \delta} W_{e}\left(\gamma^{*}\right) \\
& =\sum_{j=k^{*}}^{\infty} \phi_{e \eta}\left(\alpha, \alpha+\delta+\lambda^{j} \beta\right) \frac{\gamma^{j}}{(r+\gamma)^{j+1}}, \quad \gamma=\gamma^{*}
\end{aligned}
$$

Since $\phi_{e \eta}<0, W_{e}\left(\gamma^{*}\right)$ falls if $\delta$ rises.

If the change in $\delta$ is large, then $k^{*}$ will increase; i.e., a larger number of successful cost reductions will be necessary before entry. Further, $\alpha+\delta+\lambda^{k^{*}} \beta$ might increase or decrease, so that costs at entry time may be larger or smaller. To keep the analysis tractable, assume that when $\delta$ rises, $k^{*}$ rises to $\left(k^{*}+1\right)$ but entry-time costs $\left(\alpha+\delta+\lambda^{k^{*}} \beta\right)$ remain the same, and $\gamma^{*}$ continues to be an interior optimum. Hence $W_{e}^{\prime}\left(\gamma^{*}\left(k^{*}\right), k^{*}\right)=0$ and $W_{e}^{\prime}\left(\gamma^{*}\left(k^{*}+1\right), k^{*}+1\right)=0$. Therefore, $\gamma^{*}\left(k^{*}\right)>\gamma^{*}\left(k^{*}+1\right)$ if

$$
W_{e}^{\prime}\left(\gamma^{*}\left(k^{*}\right), k^{*}+1\right)<W_{e}^{\prime}\left(\gamma\left(k^{*}\right), k^{*}\right)=0 \text {. }
$$

Or, $\gamma^{*}\left(k^{*}\right)>\gamma^{*}\left(k^{*}+1\right)$ if $W_{e}^{\prime}$ is a decreasing function of $k^{*}$. Although $k^{*}$ is an integer, we can treat $k^{*}$ as a continuous variable to calculate $\partial W_{e}^{\prime} / \partial k^{*}$ and claim that $\gamma^{*}\left(k^{*}\right)>\gamma^{*}\left(k^{*}+1\right)$ if $\partial W_{e}^{\prime} / \partial k^{*}<0$. In the following, $\eta_{k^{*}+j}$ is kept constant for all $j=0,1, \ldots$ because of the assumption of constancy of entry-time costs (which implies constancy of costs at all subsequent stages):

$$
\begin{aligned}
& \frac{\partial W_{e}^{\prime}\left(\gamma^{*}\right)}{\partial k^{*}}=\frac{r \gamma^{k^{*}-1}}{(r+\gamma)^{k^{*}+1}}\left(1+k^{*} \log \left(\frac{\gamma}{r+\gamma}\right)\right)\left[\sum_{j=0}^{\infty} \phi_{e}\left(\alpha, \eta_{k^{*}+j}\right) \frac{\gamma^{j}}{(r+\gamma)^{j+1}}\right] \\
&+\log \left(\frac{\gamma}{r+\gamma}\right) \frac{\gamma^{k^{*}}}{(r+\gamma)^{k^{*}}} \sum_{j=0}^{\infty} \phi_{e}\left(\alpha, \eta_{k^{*}+j}\right) \frac{(j r-\gamma) \gamma^{j+1}}{(r+\gamma)^{j+2}}, \quad \gamma=\gamma^{*} .
\end{aligned}
$$

Rearranging the second term on the right-hand side of (25), we get

$$
\frac{\partial W_{e}^{\prime}\left(\gamma^{*}\right)}{\partial k^{*}}=\frac{r \gamma^{k^{*}-1}}{(r+\gamma)^{k^{*}+1}}\left(1+k^{*} \log \left(\frac{\gamma}{r+\gamma}\right)\right)\left[\sum_{j=0}^{\infty} \phi_{e}\left(\alpha, \eta_{k^{*}+j}\right) \frac{\gamma^{j}}{(r+\gamma)^{j+1}}\right]
$$




$$
\begin{array}{r}
+\log \left(\frac{\gamma}{r+\gamma}\right) \frac{\gamma^{k^{*}}}{(r+\gamma)^{k^{*}}}\left(\sum _ { j = 0 } ^ { \infty } ( j + 1 ) \gamma ^ { j } ( r + \gamma ) ^ { - j - 2 } \left[\phi_{e}\left(\alpha, \alpha+\delta+\lambda^{k^{*}+j+1} \beta\right)\right.\right. \\
\left.\left.-\phi_{e}\left(\alpha, \alpha+\delta+\lambda^{k^{*+j}} \beta\right)\right]\right), \quad \gamma=\gamma^{*} .
\end{array}
$$

As $\phi_{e \eta}<0$ and $\gamma^{*} /\left(r+\gamma^{*}\right)<1$, the second term on the right-hand side of (26) is negative. Therefore, $\partial W_{e}^{\prime}\left(\gamma^{*}\right) / \partial k^{*}<0$ if $\left[1+k^{*} \log \left(\gamma^{*} /\left(r+\gamma^{*}\right)\right)\right]<0$ or if

$$
\left(\gamma^{*} /\left(r+\gamma^{*}\right)\right)^{k^{*}}<\frac{1}{e}
$$

Therefore, if $\delta$ rises in such a way that $k^{*}$ increases but costs at entry time and at all subsequent stages remain constant, then $\gamma^{*}$ will fall if (27) is satisfied or more generally, if (26) is negative (i.e., if the total effect of a change in $k^{*}$ is a reduction in additional profit obtained from a change in $k^{*}$ ). It can be shown that (27) is satisfied only when $\gamma^{*} / k^{*}<r$ or the value of $\gamma^{*}$ is relatively low (see the remarks following Proposition 3).

If a change in $\delta$ entails both a rise in $k^{*}$ and a change in $\alpha+\delta+\lambda^{k^{*}} \beta$, then two possibilities (among many) are given below:

(a) If $\left(\lambda^{k^{*}} \beta+\delta+\alpha\right)$ rises as $\delta$ rises, (27) is satisfied, and $\phi_{e}$ is convex in $\eta$, then $\gamma^{*}$ falls as $\delta$ rises.

(b) If $\left(\lambda^{k^{*}} \beta+\delta+\alpha\right)$ falls as $\delta$ rises, (26) is negative, $\phi_{e}$ is concave in $\eta$, and (23a) and (23b) are satisfied, then $\gamma^{*}$ falls as $\delta$ rises.

Proposition 1 and equations (26) and (27) provide other possibilities.

Next, the effect of technical progress on dynamic equilibrium will be discussed. If there is disembodied technical progress whereby both the monopolist and the entrant may lower their costs, then in this model $\alpha$ falls. The effect on $\gamma^{*}$ will depend on the nature of

$$
\frac{d \phi_{e}(\alpha, \eta)}{d \alpha}=\phi_{e \alpha}+\phi_{e \eta}
$$

(because $\eta=\alpha+\delta+\lambda^{k} \beta$ ). There is no a priori reason to assume $\phi_{e \alpha}+\phi_{e \eta}$ is rising in $\alpha$ (although this is satisfied in case of straight-line demand curves). We know that

$$
\frac{d \gamma^{*}}{d \alpha}=-\frac{1}{W_{e}^{\prime \prime}\left(\gamma^{*}\right)} \frac{\partial W_{e}^{\prime}\left(\gamma^{*}\right)}{\partial \alpha} \text {. }
$$

If $\phi_{e \alpha}+\phi_{e \eta}$ is negative and rising in $\eta$, then it can be shown that $d \gamma^{*} / d \alpha<0$. Therefore, the following has been proved:

Proposition 2. Suppose technical progress reduces unit costs of both the monopolist and the entrant. Further, at higher levels of the entrant's costs, let technical progress lead to a smaller increase in postentry profits of the entrant. Then technical progress will result in a higher speed of learning, earlier expected entry time, and higher postentry profits for the entrant.

Turning to the case of a change in the rate of interest, it is seen from (19) that a rise in $r$ will reduce the present value of marginal learning costs, but it will also change the discounted value of postentry profits. Thus the effects on $\gamma^{*}$ and other variables depend on whether both effects operate in the same direction. Omitting all calculations, we state the following proposition:

Proposition 3. A rise in the rate of interest will reduce the speed of learning if $\gamma^{*} / k^{*}>r$. If $\gamma^{*} / k^{*}<r$, then the speed of learning will be reduced if the reduction of present value of marginal learning costs is greater than the reduction in present value of postentry profits. 
If the speed of learning falls, expected entry time will increase and the entry time discount factor will fall.

The condition $\left(\gamma^{*} / k^{*}\right)>r$ may be interpreted in the following way. The probability of active entry per success $\left(\gamma^{*} / k^{*}\right)$ can be regarded as an internal discount rate for the learning process. If this internal discount rate is greater than the rate of interest, a rise in $r$ will always reduce the present value of expected postentry profits. On the other hand, the effect is ambiguous if $\gamma^{*} / k^{*}<r^{3}$

Another possibility of a parametric change is a change in $\lambda$ which is the extent of cost reduction every time a success occurs. Analyzing this case in a way similar to that of an increase in $\delta$, we can demonstrate that a small increase in $\lambda$ (keeping $k^{*}$ constant) will reduce $\gamma^{*}$ if $\phi_{e}$ is convex in $\lambda$. Finally, the effect of an increase in market size may be examined in case of a straight-line demand curve. Omitting the proof, we state the following proposition. (The effect of an increase in $\bar{P}$ in (10a) is considered here.)

Proposition 4. An increase in the size of the market in case of a straight-line demand curve will increase the speed of learning, reduce expected entry time, and increase expected postentry profits of the entrant.

The propositions stated above indicate two important features of this type of equilibrium. First, the amount of uncertainty is an endogenous decision variable controlled by the entrant. If the entrant chooses $\gamma^{*}=0$, he faces no uncertainty, but he ceases to be a potential entrant if $\eta_{0}<\eta^{*}(\alpha)$. On the other hand, the higher the value of $\gamma$ chosen, the higher is the expected value of postentry profits, but the higher is the variance of per period postentry profits. Hence, $\gamma$ itself is a measure of the amount of risk taken by the entrant. Therefore, from Propositions 1-4, it is seen that (if the postentry profits satisfy the respective convexity conditions) the entrant takes a smaller risk if his cost difference with the monopolist increases, if technical progress reduces the costs of both producers, or if the rate of interest rises. On the other hand, market expansion induces the entrant to take a greater risk.

Second, plausible conclusions are obtained in this model when $\phi_{e}(\alpha, \eta)$, the entrant's postentry profit in static Cournot-Nash equilibrium, is convex (rather than concave) with respect to the entrant's unit cost $\eta$ (e.g., a rise in the initial cost difference will reduce the speed of learning if $\phi_{e}$ is convex in $\left.\eta\right)$. It should be noted, however, that $\phi_{e}(\alpha, \eta)$ is not the usual profit function but profit as a function of costs in Cournot-Nash equilibrium.

\section{Concluding remarks}

- Since learning effects are significant in many industries, ${ }^{4}$ it is important to analyze the effectiveness of different government policies to expedite entry in those industries. The results obtained in the preceding sections are helpful in this regard. First, Proposition 1 indicates that lowering the initial cost difference $\delta$ through a production cost subsidy to the entrant will increase the speed of learning and expedite entry if postentry profits are convex in the entrant's unit costs. Entry may be delayed, however, if postentry profits are concave. It is well known that the standard analytical forms of market demand functions do not always ensure convexity of profit functions in Cournot-Nash equilibrium. Hence,

${ }^{3}$ If $\gamma^{*} / k^{*}<r$ or $k^{*} / \gamma^{*}>1 / r$, then the expected entry time $k^{*} / \gamma^{*}$ is quite large. If $r=5 \%$ per year, then $k^{*} / \gamma^{*}>20$ years; if $r=3 \%$, then $k^{*} / \gamma^{*}>33.3$ years. Unless the level of $F^{\prime}(\gamma)$-marginal learning costs-is quite high for small values of $\gamma$, it is unlikely that values of $\gamma$ would be chosen to allow for entry to occur at such a late date.

${ }^{4}$ Learning effects have been empirically investigated in the airframe industry (Alchian, 1963), in nuclear power technology (Zimmerman, 1982) and in transportation equipment, farm machinery, and combustion engines (Sahal, 1981). 
unless the shape of the profit function is empirically verified to be convex, a cost subsidy to entrants may have a perverse effect. On the other hand, a direct reduction of learning costs $F(\gamma)$ may be achieved through provision of better information about markets and technologies or investment credits and will always expedite entry. Second, financial incentives in the form of lower interest rates will always increase the entrant's speed of learning if his current speed of learning is low (Proposition 3). Finally, any policy conducive to technical progress beneficial to all producers may actually delay entry (Proposition 2). In sum, it can be said that a direct reduction of learning costs will always expedite entry, whereas other policies will be successful only under special circumstances. Of course, the above policy implications will be more pertinent if this model can be extended to incorporate the possibility of several incumbents' and entrants' adopting different learning strategies simultaneously and the external effects associated with learning. Thus, this article should be seen only as a first step towards building a framework for analysis of both theoretical and policy-oriented issues related to the interaction among learning, competitive behavior, and the dynamics of industrial structure.

\section{References}

Abell, D.F. ANd Hammond, J.S. Strategic Market Planning. Englewood Cliffs, N.J.: Prentice-Hall, 1979.

Alchian, A. "Reliability of Progress Curves in Airframe Production." Econometrica, Vol. 31 (1963), pp. 679-693.

BartLetT, F.R.S. An Introduction to Stochastic Processes. London: Cambridge University Press, 1978.

Bhattacharya, G. "Learning and the Behavior of Potential Entrants." Research Papers in Economics, \#107, University of Kansas, 1982.

BuZACOTT, J. et al., EDS. Scale in Production Systems. Laxenburg: International Institute for Applied Systems Analysis, 1981.

GolD, B. "Changing Perspectives on Size, Scale, and Returns: An Interpretive Survey." Journal of Economic Literature, Vol. 19 (1981), pp. 5-33.

Karlin, S. And TaYlor, H.M. A First Course in Stochastic Processes. New York: Academic Press, 1975.

PORTER, M.E. Competitive Strategy. New York: Free Press, 1980.

SAHAL, D. Patterns of Technological Innovation. Reading, Mass.: Addison-Wesley, 1981.

SELTEN, R. "Reexamination of the Perfectness Concept for Equilibrium Points in Extensive Games." International Journal of Game Theory, Vol. 4 (1975), pp. 25-55.

SPENCE, A.M. “The Learning Curve and Competition." Bell Journal of Economics, Vol. 12 (1981), pp. 49-70.

ZIMMERMAN, M. "Learning Effects and the Commercialization of New Energy Technologies: The Case of Nuclear Power." Bell Journal of Economics, Vol. 13 (1982), pp. 297-310. 
Copyright of RAND Journal of Economics is the property of RAND Journal of Economics. The copyright in an individual article may be maintained by the author in certain cases. Content may not be copied or emailed to multiple sites or posted to a listserv without the copyright holder's express written permission. However, users may print, download, or email articles for individual use. 